\title{
PATH DERIVATIVES AND GROWTH CONTROL
}

\author{
A. M. BRUCKNER ${ }^{1}$ AND K. G. JOHNSON
}

\begin{abstract}
We show that a standard theorem relating the growth of a function on a measurable set to constraints on its Dini derivates extends to a class of generalized derivatives. More precisely, we show that if $\left|\bar{F}_{E}^{\prime}\right| \leqslant M$ and $\left|F_{E}^{\prime}\right| \leqslant M$ on a measurable set $A$, then $\lambda(F(A)) \leqslant M \lambda(A)$. Here $\lambda$ denotes Lebesgue measure and $\bar{F}_{E}^{\prime}$ and $F_{E}^{\prime}$ are the extreme derivates of $F$ relative to a system of paths which satisfy the Intersection Condition [1]. In particular, the result holds in the setting of unilateral differentiation, approximate differentiation, preponderant differentiation and qualitative differentiation.
\end{abstract}

1. Introduction. The elementary result that if a differentiable function $F$ satisfies the inequality $\left|F^{\prime}\right| \leqslant M$ on an interval $[a, b]$, then $|F(b)-F(a)| \leqslant M(b-a)$, has been generalized in many ways. For example, the interval can be replaced with an arbitrary measurable set $A$, and the differentiability requirement can be dropped. We then arrive at the following theorem: If $F$ is finite on $A$ and $\left|\bar{F}^{+}\right| \leqslant M$ on $A$, then $\lambda(F(A)) \leqslant M \lambda(A)$. (Even measurability of $A$ can be dropped if Lebesgue measure $\lambda$ is replaced with Lebesgue outer measure [3, p. 271].) This theorem and its variants give useful controls on the growth of a function in terms of constraints on the Dini derivates. An indication of the ways in which such controls are natural and useful in differentiation theory and integration theory can be seen by consulting [3 or $\mathbf{2}, \mathrm{p}$. $208 \mathrm{ff}]$.

The purpose of this paper is to prove a theorem which shows that the desired control on the growth of a function may exist even if the Dini derivates are not constrained, provided corresponding constraints are imposed on the extreme derivates relative to certain forms of generalized differentiation.

2. Preliminaries. In [1], one finds a development of an approach to differentiation which includes a number of known generalized derivatives.

Let $I_{0}$ be a fixed interval and let $x \in I_{0}$. A path leading to $x$ is a set $E_{x} \subset I_{0}$ such that $x \in E_{x}$ and $x$ is a point of accumulation of $E_{x}$. A system of paths is a collection $E=\left\{E_{x}: x \in I_{0}\right\}$ such that for every $x \in I_{0}$, the set $E_{x}$ is a path leading to $x$. A function is $E$-differentiable at $x$ with $E$-derivative $F_{E}^{\prime}(x)$ provided

is finite.

$$
F_{E}^{\prime}(x)=\lim _{\substack{y \rightarrow x \\ y \in E_{x}}} \frac{F(y)-F(x)}{y-x}
$$

Received by the editors September 9, 1982 and, in revised form, September 24, 1982.

1980 Mathematics Subject Classification. Primary 26A24; Secondary 28A15.

Key words and phrases. Generalized derivatives, path derivatives, Intersection Condition, generalized absolute continuity.

'This author was supported in part by an NSF grant. 
The extreme $E$-derivates $\bar{F}_{E}^{\prime}$ and $\underline{F}_{E}^{\prime}$ are defined in the obvious manner. A function which is $E$-differentiable at each point of a set $A$ is called $E$-differentiable on $A$; if $A=I_{0}$, it is simply called $E$-differentiable.

One finds in [1] that the behavior of an $E$-differentiable function and its $E$-derivative are very much determined by certain geometric conditions of the system $E$. One such condition will be important in the sequel.

Definition [1]. Let $E$ be a system of paths. We say that $E$ satisfies the Intersection Condition if there exists a positive function $\delta$ defined on $I_{0}$ such that if $0<y-x<\min (\delta(x), \delta(y))$, then $E_{x} \cap E_{y} \cap[x, y]$ is nonempty.

Many forms of generalized differentiation give rise to systems of paths that satisfy the Intersection Condition. This is true, for example, of unilateral differentiation, approximate differentiation, preponderant differentiation and qualitative differentiation. $($ See $[1,3.5]$.)

3. Growth theorems. We turn now to our main result.

THEOREM 1. Let $E$ be a system of paths satisfying the Intersection Condition on an interval $I_{0}$. Let $F$ be a function defined on $I_{0}$ and satisfying the inequalities $\left|\bar{F}_{E}^{\prime}\right| \leqslant M$ and $\left|\underline{F}_{E}^{\prime}\right| \leqslant M$ on a measurable set $A \subset I_{0}$. Then the set $F(A)$ is measurable and $\lambda(F(A)) \leqslant M \lambda(A)$.

Proof. Since $\bar{F}_{E}^{\prime}$ and $\underline{F}_{E}^{\prime}$ are finite on $A$, we can express $A$ as a disjoint countable union of sets $A_{k}$ on each of which $F$ is absolutely continuous, AC. (This was established in $[\mathbf{1}, 4.6]$.) The sets $A_{k}$ may be chosen measurable [3, p. 222]. It follows that $F$ satisfies Luzin's condition (N) on $A$ (so $F(A)$ is measurable) and also satisfies Banach's condition (S) on each of the sets $A_{k}$. Thus, to each positive number $\varepsilon_{k}$ corresponds a number $\delta_{k}>0$ such that if $B_{k} \subset A_{k}$ and $\lambda\left(A_{k} \backslash B_{k}\right)<\delta_{k}$ then $\lambda\left(F\left(A_{k} \backslash B_{k}\right)\right)<\varepsilon_{k}$.

Now let $\varepsilon>0$, let $\varepsilon_{k}=\varepsilon / 2^{k}$ and choose $\delta_{k}$ as above. Since $F$ is approximately differentiable a.e. on each of the sets $A_{k}$, there exist closed sets $B_{k}$ and differentiable functions $F_{k}$ such that for every $k=1,2,3, \ldots$,

(i) $B_{k} \subset A_{k}$,

(ii) $\lambda\left(A_{k} \backslash B_{k}\right)<\delta_{k}$,

(iii) $F_{k}=F$ on $B_{k}$, and

(iv) $F_{k}^{\prime}=F_{a p}^{\prime}$ a.e. on $B_{k}$.

(This follows readily from Whitney's Theorem [4].)

By $[1,4.2]$, the string of inequalities

$$
-M \leqslant \underline{F}_{E}^{\prime} \leqslant \bar{F}_{a p}^{\prime}=\underline{F}_{a p}^{\prime} \leqslant \bar{F}_{E}^{\prime} \leqslant M
$$

is valid a.e. on $B_{k}$. It now follows from the theorem stated in our introduction together with the absolute continuity of $F$ on $B_{k}$ and (iii) and (iv) above, that

(v) $\lambda F\left(B_{k}\right) \leqslant M \lambda\left(B_{k}\right)$ for every $k$.

Now let $B=\cup B_{k}$. From (v) we infer

(vi) $\lambda(F(B)) \leqslant \Sigma \lambda\left(F\left(B_{k}\right)\right) \leqslant M \Sigma \lambda\left(B_{k}\right)=M \lambda(B)$.

On the other hand, we see from (i) and (ii) that

(vii) $\lambda(F(A \backslash B))=\lambda\left(\cup F\left(A_{k} \backslash B_{k}\right)\right) \leqslant \sum \varepsilon_{k}=\varepsilon$. 
Combining (vi) and (vii), we obtain

(viii) $\lambda(F(A)) \leqslant \lambda(F(B))+\lambda(F(A \backslash B)) \leqslant M \lambda(B)+\varepsilon \leqslant M \lambda(A)+\varepsilon$.

Since $\varepsilon$ was an arbitrary positive number, the conclusion follows from (viii).

REMARK 1. We cannot drop the requirement that $E$ satisfy the Intersection Condition from the hypothesis of Theorem 1. In fact, there exists a continuous function $F$ and as system of paths $E$ such that $F$ is $E$-differentiable and $F_{E}^{\prime} \equiv 0$ on $I_{0}$, yet $F$ is not constant. One can even require that for each $x$, the path $E_{x}$ have upper density at $x$. (See the discussion following 3.1 of [1].)

REMARK 2. We needed to introduce the sets $A_{k}$ explicitly in the proof of Theorem 1 in order to use Banach's condition (S). This condition does not necessarily hold for functions which are ACG. But a function which is AC on a set does satisfy condition (S) on that set.

COROLlary 1. Theorem 1 is valid in the settings of unilateral, approximate, preponderant and qualitative differentiation.

(This follows from the fact that each of these forms of generalized differentiation gives rise to a system of paths that satisfies the Intersection Condition.)

COROLlary 2. Suppose E satisfies the Intersection Condition. Then

(i) If $F_{E}^{\prime}=0$ on a measurable set $A$, then $\lambda(F(A))=0$.

(ii) If $F_{E}^{\prime}$ is finite on a null set $A$, then $\lambda(F(A))=0$.

(iii) If $F_{E}^{\prime}$ is finite on a measurable set $A$ and vanishes a.e. on $A$, then $\lambda(F(A))=0$.

((iii) follows from (i) and the fact that $F$ is ACG on $A[1,4.6]$.)

Corollary 2(iii) admits of a converse. Suppose that $F_{E}^{\prime}$ is, say, positive on a measurable set $A, \lambda(A)>0$. Arguing as in the proof of Theorem 1 and making full use of Whitney's Theorem [4], we obtain a closed set $B \subset A(\lambda(B)>0)$ and a continuously differentiable function $G$ such that $G=F$ on $B$. It follows easily that there is an interval $I$ and a number $\delta>0$ such that $\lambda(B \cap I)>0$ and $G^{\prime} \geqslant \gamma$ on $I$.

Now $\lambda(G(B \cap I)) \geqslant \gamma \lambda(B \cap I)>0$ [2, p. 210]. Since $F$ agrees with $G$ on $B \cap I$, we conclude $\lambda(F(A)) \geqslant \lambda(F(B)) \geqslant \lambda(F(B \cap I))=\lambda(G(B \cap I))>0$.

The preceding discussion together with Corollary 2(iii) gives us a companion to Theorem 1.

THEOREM 2. Let $E$ be a system of paths satisfying the Intersection Condition. If a function $F$ is E-differentiable on the measurable set $A$, then $\lambda F(A)=0$ if and only if $F_{E}^{\prime}=0$ a.e. on $A$.

COROLlary 3. Theorem 2 applies to the forms of generalized differentiation mentioned in Corollary 1.

\section{REFERENCES}

1. A. M. Bruckner, R. J. O'Malley and B. S. Thomson, Path derivatives: a unified view of certain generalized derivatives, Trans. Amer. Math. Soc. (to appear).

2. I. P. Natanson, Theory of functions of a real variable, Vol. I, Ungar, New York, 1961.

3. S. Saks, Theory of the integral, Monograf. Mat., PWN, Warszawa-Lwow, 1937.

4. H. Whitney, On totally differentiable and smooth functions, Pacific J. Math. 1 (1951), 143-159.

Department of Mathematics, University of California, Santa Barbara, California 93106 